\title{
Comparison of the Oswestry Disability Index and Magnetic Resonance Imaging Findings in Lumbar Canal Stenosis: An Observational Study
}

\author{
Vijay G Goni ${ }^{1}$, Aravind Hampannavar ${ }^{1}$, Nirmal Raj Gopinathan ${ }^{1}$, Paramjeet Singh ${ }^{2}$, Pebam Sudesh ${ }^{1}$, \\ Rajesh Kumar Logithasan ${ }^{1}$, Anurag Sharma ${ }^{1}$, Shashidhar BK ${ }^{1}$, Radheshyam Sament ${ }^{1}$ \\ ${ }^{1}$ Department of Orthopaedics, PostGraduate Institute of Medical Education and Research, Chandigarh, India \\ ${ }^{2}$ Department of Radiology, PostGraduate Institute of Medical Education and Research, Chandigarh, India
}

\begin{abstract}
Study Design: Cross-sectional study.
Purpose: The aim of the study was to determine relationship between the degrees of radiologically demonstrated anatomical lumbar canal stenosis using magnetic resonance imaging (MRI) and its correlation with the patient's disability level, using the Oswestry Disability Index (ODI).

Overview of Literature: The relationship between the imaging studies and clinical symptoms has been uncertain in patients suffering from symptomatic lumbar canal stenosis. There is a limited number of studies which correlates the degree of stenosis with simple reproducible scoring methods.

Methods: Fifty patients were selected from 350 patients who fulfilled the inclusion criteria. The patients answered the nationallanguage translated form of ODI. The ratio of disability was interpreted, and the patients were grouped accordingly. They were subjected to MRI; and the anteroposterior diameters of the lumbar intervertebral disc spaces and the thecal sac cross sectional area were measured. Comparison was performed between the subdivisions of the degree of lumbar canal stenosis, based on the following: anteroposterior diameter (three groups: normal, relative stenosis and absolute stenosis); subdivisions of the degree of central canal stenosis, based on the thecal sac cross-sectional area, measured on axial views (three groups: normal, moderately stenotic and severely stenotic); and the ODI outcome, which was also presented in 20 percentiles.

Results: No significant correlation was established between the radiologically depicted anatomical lumbar stenosis and the Oswestry Disability scores.

Conclusions: Magnetic resonance imaging alone should not be considered in isolation when assessing and treating patients diagnosed with lumbar canal stenosis.
\end{abstract}

Keywords: Lumbar vertebrae; Low back ache; Magnetic resonance imaging scan; Radiculopathy

\section{Introduction}

Lumbar canal stenosis, one of the most common etiolo- gies of geriatric back pain and related morbidity, has also become a frequent indication for spinal surgery [1]. Narrowing of the spinal canal mainly occurs as a result of

Received Aug 2, 2012; Revised Oct 13, 2012; Accepted Oct 22, 2012

Corresponding author: Nirmal Raj Gopinathan

Department of Orthopaedics, PostGraduate Institute of Medical Education and Research,

Chandigarh-160012, India

Tel: +91-172-2756740,+91-172-2744477, Fax: 91-172-2744401, E-mail: dr.nirmalraj78@gmail.com 
degenerative bony overgrowth and soft tissue changes [2]. The diagnosis depends on various factors, including the presenting history, physical findings and imaging modalities. Magnetic resonance imaging (MRI) has been proven to be the mainstay of investigation [3]. When the conservative treatment fails, surgical decompression and stabilization are indicated. However, the results of the surgery are often inconsistent and more closely dependent on the patient's postoperative satisfaction level [4]. There are studies which support both the surgical and conservative treatment modalities $[5,6]$.

The relationship between the imaging studies and clinical symptoms has been uncertain, as there are a large number of patients with marked clinical findings with minimal imaging findings, and vice versa. Numerous studies have stated the necessity of appropriate clinical and radiological correlation for better interpretation of the treatment, be it surgical or non-surgical [7]. The Oswestry Disability Index (ODI), has proven to be a reliable method for the assessment of disability in patients with lower back pain [8]. There is a limited number of studies in the literature which correlates the degree of stenosis with simple reproducible scoring methods. The aim of this study was to determine the relationship between the degrees of radiologically demonstrated anatomical lumbar canal stenosis using MRI and to assess its correlation with the patient's disability level using ODI.

\section{Materials and Methods}

The study was conducted between December 2008 to December 2011. An approval from the Institute Ethics Committee was obtained before beginning the study. A total of 350 patients with low back pain and complaints of radicular pain and/or neurogenic claudication, numbness or weakness and aggravation due to walking, incurred over a period greater than two years, were evaluated clinically for lumbar canal stenosis. Among these, 50 patients with clinical features of lumbar canal stenosis were selected. The exclusion criteria were patients with peripheral vascular disorders, lower limb osteo-arthritis, especially hips and knees as assessed by physical examination and radiographs, polyneuropathy, degenerative scoliosis, degenerative spondylolisthesis greater than $5 \mathrm{~mm}$ determined by radiographs and previously-performed spinal surgery. All patients selected for the study underwent the following protocol. The patients were fully informed on the nature of the study and were included only after their surety of cooperation.

All patients were asked to duly answer and complete the ODI questionnaire (translated to the national language, Hindi). It contained six statements denoting levels 0 to 5 in each of the 10 sections, which were related to impairments like pain and abilities. The included impairment categories were personal care, lifting, walking, sitting, standing, sleeping, sex life, social life and travelling. In each section, the patient chose the statement that best described his/her status. If the limitation was between two levels, the higher point value was selected. The chosen statements received scores 0 to 5 , corresponding to the level indicated. The total scores can range from 0 (highest level of function) to 50 (lowest level of function). To accommodate patients who may not respond to every section, a percentage disability was calculated on the basis of the total possible points. Upon adding up all the points, the total score was divided by 50 and multiplied by 100 to calculate the percentage disability: total points $/ 50 \times 100=\%$ Disability. The percentage disability was interpreted, and all patients were grouped accordingly: as minimally disabled for $0 \%$ to $20 \%$; moderate disability for $21 \%$ to $40 \%$; severe disability for $41 \%$ to $60 \%$; crippled for $61 \%$ to $80 \%$; and bedridden for $81 \%$ to $100 \%$.

All patients underwent a non-contrast lumbo-sacral spinal MRI scan. The MRI scans included sagittal and axial T1- and T2-weighted images from the first lumbar to first sacral level. Spinal canal anteroposterior (AP) diameter and thecal sac cross sectional area (CSA) were measured using the sagittal and axial sections of all lumbar intervertebral levels (Figs. 1-3). All patients were

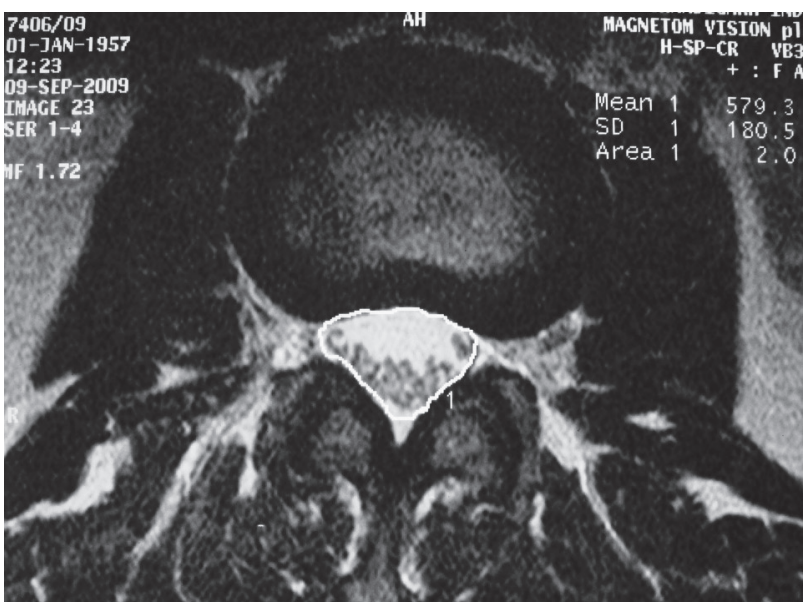

Fig. 1. Image showing a normal lumbar thecal sac area measuring 200 $\mathrm{mm}^{2}$ at L1-L2 level. 
divided into 3 groups according to the following criteria, using the smallest diameter measured among all levels. AP diameter of less than $10 \mathrm{~mm}$ was considered as absolute stenosis; 10 to $13 \mathrm{~mm}$ as relative stenosis; and more than $13 \mathrm{~mm}$ as normal [9]. Cross sectional area of less than $76 \mathrm{~mm}^{2}$ was considered as severe stenosis; 76 to 100

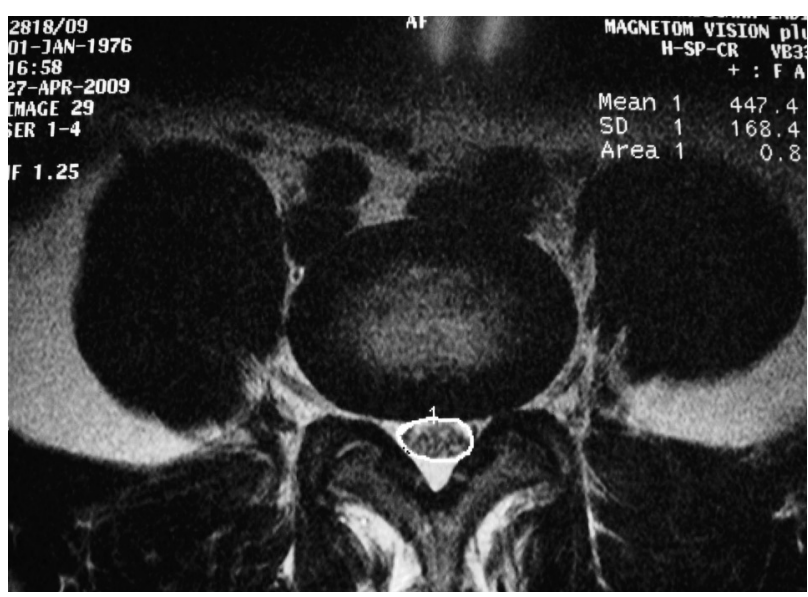

Fig. 2. Magnetic resonance image showing thecal sac measuring 80 $\mathrm{mm}^{2}$ at L5-S1 level.

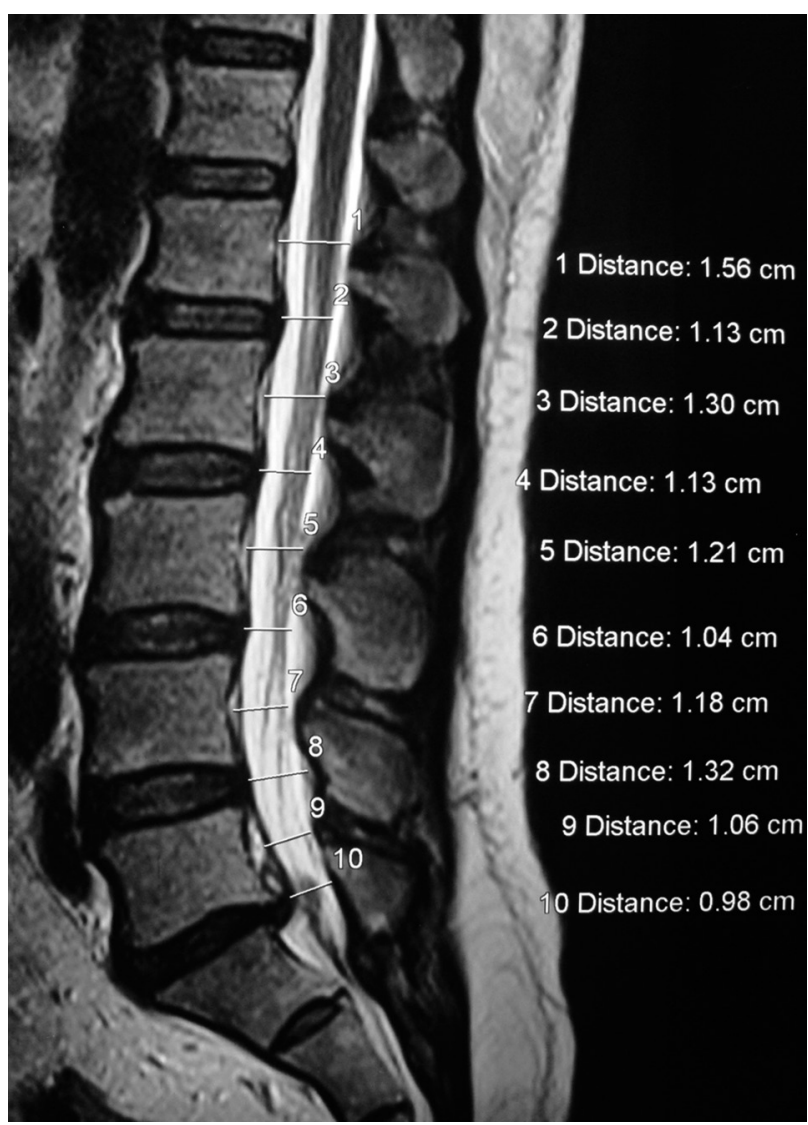

Fig. 3. Sagittal view of $L-S$ spine showing absolute stenosis at L5-S1 measuring $0.98 \mathrm{~mm}$. $\mathrm{mm}^{2}$ was considered as moderately stenotic; and greater than $100 \mathrm{~mm}^{2}$ as normal [10]. Analysis of the data was done to compare the radiological findings as recorded by the magnetic resonance imaging and the percentage disability recorded by the Oswestry Disability index. All patients were also divided into two groups, based on thecal sac CSA of $70 \mathrm{~mm}^{2}$. The disability scores of the two groups were analyzed statistically to determine the critical threshold of lumbar canal stenosis.

\section{Results}

In this study, fifty patients with clinical features of lumbar canal stenosis were selected. The average age of the patients in the study was 46 years, with the range of 28 to 64 years, with a slight female preponderance ( 27 women, 23 men). All 50 patients completed the ODI questionnaire. Each questionnaire had 10 questions correlating to 500 possible responses. Of these, 455 responses were received, giving an overall response rate of $91 \%$. There was no response given to the sections on sex life, traveling and sleeping by 41,3 and 1 of the 50 patients, respectively. In our study, the response rate for sex life was $18 \%$, traveling was $94 \%$ and sleeping was $98 \%$. The maximum number of patients responded as level 3 (in a range of $0-5$ ). If the higher levels of disability (i.e., 3, 4 or 5) were grouped together, 32, 30, 30, and 26 of the total number of patients (50) had the higher levels of disability in standing, walking, social life and lifting, respectively.

The percentage disability was interpreted, and all patients were grouped accordingly: as minimally disabled for $0 \%$ to $20 \%$; moderate disability for $21 \%$ to $40 \%$; severe disability for $41 \%$ to $60 \%$; crippled for $61 \%$ to $80 \%$; and bedridden for $81 \%$ to $100 \%$. On the basis of the percentage disability score of the ODI, out of the 50 patients, were the following groups: 3 patients demonstrated mild disability; 15 patients showed moderate disability; 23 patients had severe disability; 6 patients were crippled; and 3 patients were bedridden. Of the 50 patients, a total of 250 intervertebral spaces were analyzed for the stenotic findings. The AP diameter at the level of all 5 intervertebral spaces varied from 5 to $15.8 \mathrm{~mm}$. The most affected level was L5-S1, with the average AP diameter of 10.4 $\mathrm{mm}$. Absolute stenosis was seen in $42 \%$ of the patients, and $58 \%$ had relative stenosis on the basis of criteria as shown in annexure. The CSA of the thecal sac was also measured. The cross-sectional area of the dural sac at all 
Table 1. Anteroposterior diameter versus ODI

\begin{tabular}{lrrr} 
ODI score & $\begin{array}{r}\text { Absolute } \\
\text { stenosis }\end{array}$ & $\begin{array}{r}\text { Relative } \\
\text { stenosis }\end{array}$ & Total \\
\hline Minimal & $1(33.3)$ & $2(66.7)$ & 3 \\
Moderate & $7(46.7)$ & $8(53.3)$ & 15 \\
Severe & $9(39.1)$ & $14(60.9)$ & 23 \\
Crippled & $3(50.0)$ & $3(50.0)$ & 6 \\
Bedridden & $1(33.3)$ & $2(66.7)$ & 3 \\
Total & $21(42.0)$ & $29(58.0)$ & 50 \\
\hline
\end{tabular}

Values are presented as number (\%).

ODI, Oswestry Disability Index.

Table 2. Thecal sac CSA versus ODI

\begin{tabular}{lcccr} 
ODI score & $\begin{array}{c}\text { Severe } \\
\text { stenosis }\end{array}$ & $\begin{array}{c}\text { Moderate } \\
\text { stenosis }\end{array}$ & Normal & Total \\
Minimal & $1(33.3)$ & $1(33.3)$ & $1(33.3)$ & 3 \\
Moderate & $3(20.0)$ & $9(60.0)$ & $3(20.0)$ & 15 \\
Severe & $7(30.4)$ & $7(30.4)$ & $9(39.1)$ & 23 \\
Crippled & $3(50.0)$ & $1(16.7)$ & $2(33.3)$ & 6 \\
Bedridden & $1(33.3)$ & $1(33.3)$ & $1(33.3)$ & 3 \\
Total & $15(30.0)$ & $19(38.0)$ & $16(32.0)$ & 50 \\
\hline
\end{tabular}

Values are presented as number (\%).

CSA, cross sectional area; ODI, Oswestry Disability Index.

Table 3. ODI scores versus thecal sac CSA using $70 \mathrm{~mm}^{2}$ as cut off

\begin{tabular}{lccc} 
ODI score & $<70 \mathrm{~mm}^{2}$ & $>70 \mathrm{~mm}^{2}$ & Total \\
Minimal & $1(33.3)$ & $2(66.7)$ & 3 \\
Moderate & $1(6.7)$ & $14(93.3)$ & 15 \\
Severe & $5(21.7)$ & $18(78.3)$ & 23 \\
Crippled & $3(50.0)$ & $3(50.0)$ & 6 \\
Bedridden & $1(33.3)$ & $2(66.7)$ & 3 \\
Total & 11 & 39 & 50 \\
\hline
\end{tabular}

Values are presented as number (\%).

ODI, Oswestry Disability Index; CSA, cross sectional area.

levels varied between $245 \mathrm{~mm}^{2}$ and $17 \mathrm{~mm}^{2}$, at the level of all the intervertebral spaces. The most affected level was again L5-S1. Of the 50 patients, 15 revealed moderate and 23 revealed severe central stenosis at this level.

\section{Correlation analysis}

1) Correlation between ODI scores and AP diameter Comparison was performed between the subdivisions of the degree of lumbar canal stenosis based on the AP diameter (three groups: normal, relative stenosis and absolute stenosis) and the ODI outcome, which was also presented in 20 percentiles. Out of the 50 patients, 21 had absolute stenosis, and 13 patients among them had ODI scores indicating the severe, crippled and bedridden groups combined together. Pearson chi-square test was performed, and the $p$-value was 0.968 . It indicates that there is no statistically significant correlation between the disability levels by the ODI classification and AP diameter classification. The data is shown in Table 1.

2) Correlation between ODI scores and thecal sac CSA Comparison was also performed between the subdivisions of the degree of central canal stenosis based on the thecal sac CSA measured on axial views (three groups: normal, moderately stenotic and severely stenotic) and the ODI outcome, which was also presented in 20 percentiles (Tables 2, 3). Of the 50 patients, $30 \%$ had severe stenosis with CSA less than $76 \mathrm{~mm}^{2}$, and $38 \%$ had moderate stenosis. On the statistical analysis, there was no significant relationship between the disability level distribution by the ODI classification and the thecal sac CSA classification ( $p=0.716$ ). However, when patients were divided into two groups based on the thecal sac CSA of $70 \mathrm{~mm}^{2}$ and the disability scores of the two groups were analyzed statistically to determine the critical threshold of lumbar canal stenosis, the correlation was found to be statistically significant $(p=0.026)$.

\section{Discussion}

Lumbar spinal stenosis is defined as a narrowing of the neural canal and foramina to an extent which results in a compression of the lumbo-sacral nerve roots or cauda equina syndrome. As the articulating facets and supportive ligaments undergo degenerative hypertrophy and the osteophytic ridges form, they encroach on the neural structures passing through or the exiting the spinal canal [11]. Although the prevalence of spinal stenosis is difficult to determine as no population-based studies have as yet been done, the disorder is relatively common and has been recognized more frequently since the introduction of the advanced radiographic imaging techniques. The prevalence of the degenerative spine disease will increase with the aging of the population. Since the degree of the constriction of the spinal canal considered to be symptomatic for lumbar canal stenosis is unclear, a thorough 
understanding of the anatomic relationship of the structures within the spinal canal and the diagnostic accuracy of the imaging techniques, findings from the clinical examination and the appropriate outcome measures need to be identified to ensure accurate diagnosis and appropriate surgical intervention.

In our study, a total of 50 patients were included. The mean age of our patients was 46 years with the range of 28 to 64 years. Although the mean age is younger compared to most studies, the maximum number of patients were in the above-50-years group. Most previous studies have reported a higher mean age except the one study by Lohman et al. [12]. The relatively low age of our study population likely indicates that the patients with congenitally narrow canals may be overrepresented compared to the general population. There was a slight female preponderance in our study, with 27 out of 50 patients being female. This is in accordance with the previous studies. The average duration of pain at presentation in our study was about 3 years 4 months. The mean duration in the previous studies has ranged up to 7 years.

In our study, the ODI questionnaire (translated to Hindi) was easily comprehended and had a response rate of $91 \%$. Various studies have reported that this short, self-administered questionnaire is reproducible, reliable, internally consistent and valid and is an adequately useful instrument for the assessment of disability in patients with lower back pain. According to the walking capacity question in the questionnaire, $6 \%$ of the patients were limited to bed, and $34 \%$ had to use a walking-stick. This indicates that overall, $40 \%$ of the patients had maximum difficulties with walking up to 100 metres for which they consulted a doctor. Zeifang et al. [13] have concluded that the MRI findings seem to have less clinical relevance on the walking distance in patients with symptomatic lumbar spinal stenosis than previously assumed.

Degenerative spinal stenosis most commonly affects the L3-L4 and L4-L5 segments to cause cauda equina compression. However in our study, the maximum affected level was L5-S1 with the average antero-posterior diameter of $10.4 \mathrm{~mm}$ and the thecal sac CSA of $88 \mathrm{~mm}^{2}$. The correlation between spinal stenosis and clinical symptoms has been the subject of continuing controversy. While some authors acknowledge a correlation only for certain groups of patients, others have reported an influence of the spinal canal dimensions in multilevel foraminal narrowing $[6,12]$. None of these studies demonstrated a clear association between the degree of narrowing and clinical symptoms nor could the cutoff values be determined.

In our study, the comparison was initially performed between the subdivisions of the degree of central canal stenosis based on the AP diameter and the ODI outcome, which was presented in five categories based on severity. This comparison showed no correlation. Moreover, upon statistical evaluation of the subdivisions of the degree of central canal stenosis based on the thecal sac CSA versus the ODI percentage scores, again no significant correlation was established. Thus, no significant correlation could be established in our study between the radiologically depicted anatomical lumbar stenosis and the Oswestry Disability scores. The results in our study indicate that unclear, confusing clinical findings resembling spinal stenosis are also relatively common in patients who have mild or no narrowing of the spinal canal on the imaging. This is in accordance with the previous studies which have also noted a lack of correlation between the radiographically detected stenosis and clinical findings, and the presence or absence of the symptoms and signs [1216].

The lack of a relationship between pathophysiology and ODI scores suggests that the disability and pain in lumbar canal stenosis is determined by multiple factors. Although numerous factors have been implicated in the experience of pain in younger adults, the impact of pain on older adults has received less attention. A recent study by Weiner et al. [17] reported that medical co-morbidities and radiographic pathology were of little utility in predicting function in the older adults with persistent low back pain. Geisser et al. [15] in their study have suggested that the pain intensity was significantly associated with the perceived disability but not with the objective measures of function (walking). They also indicated that the perception of pain limits activity, or that the expected level of pain in performing an activity is more highly predictive of the functional limitations in older adults compared with the observed activity. Although this suggests that the perceived disability as measured by ODI is a better predictor, our study indicates that even the perceived disability as measured by ODI is not predictive of the underlying patho-morphology.

A range of morphologic and psychosocial variables may also play a role. Hazard et al. [18] have shown that both the psychologically disturbed and depressed patients have higher Oswestry scores than the normal patients, when 
the self-reported disability is compared with the objective physical measurements. This may explain the high ODI scores of some of our patients, who did not correspond to the degree of radiologically-shown spinal stenosis.

Spinal canal narrowing is considered to cause more problems when it occurs at several levels. In our study, the multilevel narrowing with an area of less than 100 $\mathrm{mm}^{2}$ was seen in $22 \%$ of the patients. According to the studies by Porter and Ward [19], the correlation between the radiologic and clinical findings is more obvious in the multilevel spinal narrowing compared to the single level spinal stenosis. However, we could not find any correlation between the number of levels with spinal stenosis (neither absolute nor relative) and the disability levels. The difference in the results is most likely explained by a difference in the study population.

The difficulties associated with finding such correlations include the presence of a large number of patients with spinal narrowing and a complete lack of symptoms, variations in the canal size throughout the population and a lack of an accepted system for quantifying the degree of narrowing [20]. Patients can have changes on the MR imaging compatible with the morphological diagnosis of spinal stenosis, but be asymptomatic. Another question is whether the reverse situation may occur, and if so, whether it is caused by different susceptibility of the nerves for narrowing of the spinal canal or by dynamic stenosis. The extent of narrowing is also dynamic and is likely to change with the posture of the patient. For example, extension significantly decreases the canal area, whereas flexion has the opposite effect. Therefore, a static image of the canal dimensions may not be predictive of a patient's symptoms. The lack of correlation in our study may also be related to the observation that the symptoms tend to fluctuate considerably over time, and that there is a wide variability in the lumbar dimensions among the patients who do not have clinical spinal stenosis [21].

Yukawa et al. [22] suggested that a CSA of the dural sac below $70 \mathrm{~mm}^{2}$ sharply increases the likelihood of the clinical symptoms of lumbar stenosis. The present study found that the ODI disability levels correlated significantly with the thecal sac CSA less than $70 \mathrm{~mm}^{2}$. This indicates that clinical symptoms of lumbar canal stenosis and its imaging findings correlate more significantly when stenosis is of a severe grade. This would be consistent with the physical compression of the neural elements as a pathophysiology of neurogenic claudication and is supported by several experimental studies. A study by Delamarter et al. [23] found that motor and sensory deficits may develop with $50 \%$ or greater constriction of the CSA of the spinal canal. In addition, a cadaveric study by Schonstrom and Hansson [10] demonstrated that the critical size for an increase in pressure among the nerve roots occurs when the dural sac is constricted to a CSA of less than $77 \mathrm{~mm}^{2}$.

The strengths of this study are the inclusion of a standardized technique to analyze the imaging studies and the use of a validated patient-oriented outcome scale to quantify the patient disability. Regarding the patient population in the present study, we examined a sample of patients with a relatively homogenous set of symptoms which were most characteristic of lumbar canal stenosis. This allowed us to examine and compare patients with varied canal sizes. However, only a limited subset of patients had severe lumbar canal stenosis defined by the anthropomorphic criteria. The results may differ if the patient population was a subset of patients selected for surgery. The fact that in some patients the radiological changes were more extensive than as expected from the clinical picture and that the degree of narrowing did not correspond to the severity of ODI percentage disability further establishes that lumbar canal stenosis is a clinicoradiological syndrome.

\section{Conclusions}

Magnetic resonance imaging alone should not be considered in isolation when assessing and treating patients diagnosed with lumbar canal stenosis. The future research should be directed in tailoring a dynamic assessment modality to quantify spinal canal stenosis.

\section{Conflict of Interest}

No potential conflict of interest relevant to this article was reported.

\section{References}

1. Deyo RA, Gray DT, Kreuter W, Mirza S, Martin BI. United States trends in lumbar fusion surgery for degenerative conditions. Spine (Phila Pa 1976) 2005;30:1441-5.

2. Yong-Hing K, Kirkaldy-Willis WH. The pathophysi- 
ology of degenerative disease of the lumbar spine. Orthop Clin North Am 1983;14:491-504.

3. Fritz JM, Delitto A, Welch WC, Erhard RE. Lumbar spinal stenosis: a review of current concepts in evaluation, management, and outcome measurements. Arch Phys Med Rehabil 1998;79:700-8.

4. Verbiest H. Results of surgical treatment of idiopathic developmental stenosis of the lumbar vertebral canal: a review of twenty-seven years' experience. J Bone Joint Surg Br 1977;59:181-8.

5. Herno A, Airaksinen O, Saari T. Long-term results of surgical treatment of lumbar spinal stenosis. Spine (Phila Pa 1976) 1993;18:1471-4.

6. Amundsen T, Weber H, Nordal HJ, Magnaes B, Abdelnoor M, Lilleas F. Lumbar spinal stenosis: conservative or surgical management?: a prospective 10year study. Spine (Phila Pa 1976) 2000;25:1424-35.

7. Arbit E, Pannullo S. Lumbar stenosis: a clinical review. Clin Orthop Relat Res 2001;(384):137-43.

8. Herno A, Airaksinen O, Saari T. Computed tomography after laminectomy for lumbar spinal stenosis. Patients' pain patterns, walking capacity, and subjective disability had no correlation with computed tomography findings. Spine (Phila Pa 1976) 1994;19:19758.

9. Ullrich CG, Binet EF, Sanecki MG, Kieffer SA. Quantitative assessment of the lumbar spinal canal by computed tomography. Radiology 1980;134:137-43.

10. Schonstrom N, Hansson T. Pressure changes following constriction of the cauda equina: an experimental study in situ. Spine (Phila Pa 1976) 1988;13:385-8.

11. Ciricillo SF, Weinstein PR. Lumbar spinal stenosis. West J Med 1993;158:171-7.

12. Lohman CM, Tallroth K, Kettunen JA, Lindgren KA. Comparison of radiologic signs and clinical symptoms of spinal stenosis. Spine (Phila Pa 1976) 2006;31:1834-40.

13. Zeifang F, Schiltenwolf M, Abel R, Moradi B. Gait analysis does not correlate with clinical and MR imaging parameters in patients with symptomatic lumbar spinal stenosis. BMC Musculoskelet Disord 2008;9:89.

14. Athiviraham A, Yen D, Scott C, Soboleski D. Clinical correlation of radiological spinal stenosis after standardization for vertebral body size. Clin Radiol 2007;62:776-80.

15. Geisser ME, Haig AJ, Tong HC, et al. Spinal canal size and clinical symptoms among persons diagnosed with lumbar spinal stenosis. Clin J Pain 2007;23:7805.

16. Sirvanci M, Bhatia M, Ganiyusufoglu KA, et al. Degenerative lumbar spinal stenosis: correlation with Oswestry Disability Index and MR imaging. Eur Spine J 2008;17:679-85.

17. Weiner DK, Rudy TE, Kim YS, Golla S. Do medical factors predict disability in older adults with persistent low back pain? Pain 2004;112:214-20.

18. Hazard RG, Bendix A, Fenwick JW. Disability exaggeration as a predictor of functional restoration outcomes for patients with chronic low-back pain. Spine (Phila Pa 1976) 1991;16:1062-7.

19. Porter RW, Ward D. Cauda equina dysfunction. The significance of two-level pathology. Spine (Phila Pa 1976) 1992;17:9-15.

20. Szpalski M, Gunzburg R. Lumbar spinal stenosis in the elderly: an overview. Eur Spine J 2003;12 Suppl 2:S170-5.

21. Amundsen T, Weber H, Lilleas F, Nordal HJ, Abdelnoor M, Magnaes B. Lumbar spinal stenosis: clinical and radiologic features. Spine (Phila Pa 1976) 1995;20:1178-86.

22. Yukawa Y, Lenke LG, Tenhula J, Bridwell KH, Riew $\mathrm{KD}$, Blanke K. A comprehensive study of patients with surgically treated lumbar spinal stenosis with neurogenic claudication. J Bone Joint Surg Am 2002;84:1954-9.

23. Delamarter RB, Bohlman HH, Dodge LD, Biro C. Experimental lumbar spinal stenosis: analysis of the cortical evoked potentials, microvasculature, and histopathology. J Bone Joint Surg Am 1990;72:110-20. 